\title{
L'ÉCO-CITOYENNETÉ AU RISQUE DE LA MANIPULATION
}

\author{
Anne-Hélène Le Cornec Ubertini ${ }^{1}$
}

La question de savoir si l'homme est libre ou non a sans doute son intérêt, mais c'est en métaphysique qu'elle a sa place et les sciences positives peuvent et doivent s'en désintéresser.

Emile Durkheim, Cours de science sociale. Leçon d'ouverture

La question du libre arbitre de l'homme n'est toujours pas résolue et Émile Durkheim avait raison de dire à ses étudiants qu'il ne fallait pas attendre la solution pour avancer. Arriverons-nous jamais à mesurer ce qui relève de notre consentement libre et éclairé ? Certes les philosophes ont répondu à la question, chacun à sa manière. L'existentialisme de Jean Paul Sartre (1945) par exemple, pourrait clore le débat en affirmant que nous avons toujours le choix or cette idée de libre choix bute selon nous sur la question de la manipulation même si là encore nous avons le choix de nous préparer à lutter contre la manipulation. La logique sartrienne est imparable sauf à dire qu'il s'agit d'un idéal et que la réalité est plus complexe. Les sciences positives sont-elles, comme le pense Durkheim, à cause de leur approche complexe ou proximale, incapables de traiter cette thématique alors que seule la métaphysique, par sa visée distale et moins contingente, pourrait l'envisager

1 Maître de conférences en Sciences de l'Information et de la Communication. I3M «Information, Milieux, Médias et Médiations », UNS/USTV

Recherches en communication, $\mathrm{n}^{\circ} 28$ (2007). 
? Nous ne le pensons pas car les temps changent. Notre propos n'est donc pas d'aborder ce thème sous l'angle métaphysique mais d'essayer de montrer que les sciences positives, comme les sciences sociales, peuvent s'en emparer et éclairer l'opinion avec pertinence. À partir de récents travaux sur la formation au développement durable, nous voulons proposer des pistes de réflexion sur la manière dont les notions du consentement et de la liberté traversent actuellement la société et soulèvent selon nous de vives interrogations.

\section{Passage à l'acte}

L'homme et sa planète sont en péril, il nous faut donc adopter des comportements différents, moins polluants, pour vivre tous mieux et ne pas hypothéquer les chances de survie des générations futures. La cause semble entendue même si ici et là de farouches opposants à une telle mise à mal de notre liberté de produire et de consommer se font entendre. Si nous sommes largement favorables à la protection de l'environnement, de la parole aux actes il y a plus qu'un pas à franchir. Une longue tradition de travaux notamment en psychologie sociale sur le passage des idées, ou attitudes, aux actes tend à démontrer que les comportements ne sont pas toujours liés aux attitudes. La première étude fut menée en 1934 par Richard LaPiere qui observa l'attitude de deux cent cinquante hôteliers et restaurateurs vis-à-vis de deux étudiants chinois. Il voulait vérifier la consonance entre les attitudes qu'il supposait racistes et les comportements en face-à-face. Le couple de chinois n'eut qu'une réaction de rejet à déplorer. De retour dans son laboratoire, LaPiere envoya une lettre dans chaque établissement pour demander si leurs dirigeants accepteraient d'accueillir un couple de chinois. Cette fois la quasi-totalité, quatre-vingt-douze pour cent, des réponses obtenues fut négative. Donc les comportements n'étaient pas prédictifs de l'attitude. Les études qui se sont succédées jusqu'à maintenant n'ont pas réussi à démontrer de relation étroite entre attitude et comportement. Comment obtenir plus de cohérence ? Kurt Lewin en 1947 a été le premier à comprendre les principaux mécanismes de l'engagement puis Charles Kiestler en 1971 a développé sa théorie : certains actes anodins nous engagent inconsciemment dans des comportements futurs beaucoup plus lourds de conséquences. En France, Jean-Léon Beauvois et Robert-Vincent Joule, à partir de 1987, ont parfaitement illustré ces questions. Ils détaillent les techniques pour engager une personne dans son acte. Obtenir par exemple un premier comportement peu coûteux 
comme « donner » l'heure permet d'obtenir, de la même personne, dans un deuxième temps un comportement beaucoup plus coûteux sans qu'il se rende compte que son accord a été forcé. C'est le «pied-dans-laporte $»$.

\section{Pédagogie de l'engagement éco citoyen}

Si nous connaissons les conditions nécessaires pour engager autrui, nous pouvons obtenir de lui les comportements que nous souhaitons, sans qu'il en prenne conscience. Cela s'appelle manipuler. La personne faisant autorité obtient de manière détournée un changement de comportement et d'opinion, c'est la raison pour laquelle nous disons « engager autrui » et pas « autrui s'engage ». Et si c'était pour la bonne cause n'y aurait-il pas lieu de se laisser tenter? Après tout, qu' est-ce que manipuler si nous engageons nos concitoyens à faire le bon éco geste, est-ce manipuler s'il s'agit de renforcer la fierté de nos concitoyens et finalement est-ce manipuler dès lors que nous ne sommes pas libres. Manipuler un individu déjà manipulé, n'est-ce pas le rendre libre ? En effet, en entrant dans la société de l'information, nous sommes entrés dans la société de la manipulation où la multiplication des médias augmente proportionnellement la désinformation et la manipulation. Les résultats obtenus par Robert-Vincent Joule en matière d'éco citoyenneté depuis $2004^{1}$ jusqu'à aujourd'hui montrent l'efficacité de la manipulation :

Une huitième et dernière expérience, réalisée par Joule et commanditée par l'ADEME (ex-AFME), avait pour objectif de réduire la consommation d'électricité d'un établissement hospitalier de la région marseillaise. L'utilisation de techniques d'engagement comme le pied-dans-la-porte, l'amorçage et l'engrenage (Joule et Beauvois, 2002) a permis d'économiser plus de $20 \%$ d'électricité dans cet établissement. Economie constatée par les agents d'EDF après relevé des compteurs d'électricité.

R. V. Joule plaide pour la manipulation d'une part parce qu'elle augmente les actes citoyens donc les comportements souhaités et d'autre

1 Joule Robert Vincent, 2004, Colloque écocitoyenneté : des idées aux actes favorables à l'environnement - Recueil des interventions, p. 45-46, en ligne sur (page consultée le 8 mai 2008) :

http://www.ademe.fr/paca/images/pdf/ActesEcocit2004.pdf 
part, parce que les gens manipulés ne peuvent expliquer leurs actes que par leur personnalité. N'ayant pas conscience de la manipulation, les gens établissent ou renforcent le lien entre leurs actes citoyens et leurs traits de caractère. Pourquoi les priver de la fierté d'être quelqu'un de bien ? « Pourquoi ne pas aider les gens à tirer leur niveau d'identification de l'action vers le haut? » demande Joule (2006) ${ }^{1}$.

Allant plus loin, Joule propose de généraliser aux adultes les principes de naturalisation et de dénaturalisation qu'il préconise pour les enfants. Un acte attendu, ici un acte éco citoyen, sera « naturalisé » s'il est accompagné d'un jugement du type « cela te ressemble bien » porté par un évaluateur qui permet d'établir un lien entre le comportement d'un individu et sa personnalité. Al'inverse un acte réprouvé, ici un acte néfaste pour le développement durable, sera « dénaturalisé » en disant « cela ne te ressemble pas » pour casser le lien entre le comportement de l'individu et sa personnalité. L'évaluateur est une personne faisant autorité, le parent, l'enseignant, le chercheur, le médecin, le supérieur hiérarchique, l'expert, ... La dénaturalisation doit, toujours selon notre auteur ${ }^{2}$, bien sûr, être suivie d'une promesse de mieux faire sous forme de contrat d'action.

Il s'agit d'une pédagogie de la responsabilisation dans la mesure où ces décisions et ces engagements sont obtenus dans un climat de liberté propice à l'intériorisation des valeurs recherchées.

Comment ne pas se laisser séduire par des méthodes performantes sur le terrain, des principes simples ? Nous aurions enfin trouvé ce Graal poursuivi par Taylor au début de l'ère industrielle, le "one best way » mais dans un autre domaine, celui du passage à l'acte éco citoyen. Voie royale et unique contre diversité et concurrence des approches. L'alternative la plus évidente serait de faire appel à la raison des citoyens mais cela ne produit pas les effets attendus, les attitudes et les actes restent trop dissociés, par exemple les automobilistes ne ralentissent plus dès que le gendarme disparaît alors qu'ils partagent l'idée qu'il faut être prudents au volant. Puisque la raison ne suffit pas à adopter des comportements citoyens et puisque la manipulation n'a

1 Conférence en ligne sur Canal U, (page consultée le 8 mai 2008), http://www.canal-u.education.fr/canalu/producteurs/les_amphis_de_france_5/ dossier_programmes/sciences_innovation_societe/sommes_nous_manipules

2 Joule Robert Vincent, 2004/2005, Matière à réflexion, SYMBIOSES, n 65. 
que des effets positifs telle qu'elle nous est présentée, pourquoi ne pas adhérer ? Il est toutefois difficile d'oublier que la volonté de faire le bonheur des gens malgré eux sous-tend rarement un dessein démocratique mais plutôt un système privatif de liberté. Malgré l'accent porté sur l'individu, sa responsabilité, sa personnalité, la technique souffre d'un défaut originel : l'absence de consentement des citoyens. Faire porter à l'individu seul la responsabilité de ce qui lui arrive, de ce qu'il fait, correspond certes à une norme sociale, la norme "d'internalité » issue du choix de société que nous avons fait entre holisme et individualisme. Nous avons choisi de dire que l'homme est responsable de son destin. Les circonstances atténuantes existent mais ne font que minorer la sanction sociale formelle et informelle. Ici ce qui dérange, c'est le paradoxe, la manipulation par la naturalisation et la dénaturalisation tend à renforcer la responsabilité individuelle tout en emprisonnant l'identité des individus entre les mains de l'évaluateur. Même si nous ne condamnons pas le principe de réalité, la promotion de la manipulation, quand bien même elle n'entraînerait que des conséquences positives, nous dérange philosophiquement à la fois parce que les conséquences psychologiques et sociales à long terme n'ont pas été étudiées et surtout parce que personne ne semble s'émouvoir de cette manipulation présentée comme une forme de fraternité.

Alors il nous semble indispensable de quitter le «terrain », notamment celui de l'éco citoyenneté, pour essayer de creuser la question fondamentale de la liberté et du consentement. Quelle liberté avons-nous, quelle conscience de son éventuelle absence ? Comment sommes-nous organisés, quel contrôle social exerçons-nous sur ce consentement?

\section{Quelle liberté ?}

\section{Liberté individuelle : la part de l'inconscient}

Depuis 1888, date de la leçon d'ouverture du cours d'Émile Durkheim sur la science sociale, nos connaissances en matière de libre arbitre ont augmenté en mettant malheureusement fin à quelques illusions. Notre consentement s'obtient aisément grâce à des tours et des détours accessibles à tous les honnêtes gens, il s'obtient sans force déployer, comme par magie en nous laissant l'impression de maîtriser nos pensées et nos actes. Nous en avons donné quelques exemples plus haut. La délicieuse expression de « soumission librement consentie » inventée par Robert-Vincent Joule et Jean-Léon Beauvois (1998) 
associe l'impensable : la soumission et la liberté du consentement. Il ne s'agit pas de constater le libre choix de se soumettre mais l'effet de soumission que l'impression de consentir librement provoque. Nous donner le sentiment de liberté de choix nous conduirait à nous ligoter davantage. Ainsi, lorsque nous sommes sollicités par un tiers, le simple fait de nous signifier notre totale liberté d'accepter double les chances de notre interlocuteur que nous répondions par l'affirmative. Une idée qui prolonge l'intuition d'Aldious Huxley (1946, p. 15) sur l'efficacité de futurs dirigeants ou éducateurs n'ayant plus à contraindre des sujets esclaves puisqu'ils auraient l'amour de leur servitude.

Un gouvernement vraiment «efficient» serait celui dans lequel le
tout-puissant comité exécutif des chefs politiques et leur armée
de directeurs auraient la haute main sur une population d'esclaves
qu'il serait inutile de contraindre, parce qu'ils auraient l'amour
de leur servitude. La leur faire aimer.

Stanley Milgram (1974) finit de nous désespérer en nous prouvant que nous sommes capables de consentir à nuire gravement à notre prochain malgré l'absence de moyens de coercition réels. Souvenonsnous de cette fameuse expérience où les sujets devaient infliger des chocs électriques de plus en plus intenses à d'autres sujets lorsque leurs réponses à un questionnaire étaient fausses. Les victimes étaient des acteurs et ne recevaient pas réellement ces chocs mais les sujets «bourreaux » ne le savaient pas, ce qui n'a pas empêché plus des deux tiers d'entre eux d'infliger des chocs mortels. Milgram explique ce comportement par un «état agentique » auquel nous ne pourrions pas échapper face à un évaluateur ou un supérieur hiérarchique. Heureusement, ces troubles du consentement ne se vérifient pas dans cent pour cent des cas, nous pouvons donc espérer faire partie de la marge, des irréductibles à un statut de sujet mineur ou d'objet tout court. Maigre consolation pour qui ne jouit pas d'une confiance aveugle en lui-même.

A ces erreurs de jugement d'un processeur central dont les performances doivent aussi faire les déficiences (je répugne à penser qu'il n'y a pas une très bonne raison à tout cela), il faut ajouter notre soumission aux normes sociales. 


\section{Normes sociales}

Il n'est pas certain que notre respect des normes sociales se fasse en toute connaissance de cause. L'apprentissage des règles de nos différents groupes d'appartenance a pu se réaliser sans heurt au point que nous avons intégré la plupart d'entre elles sans conscience qu'il s'agissait de normes. Nous sommes alors dans un cas de figure qui nous rapproche des constats précédents. Nous suivons par conformisme sans le savoir. Selon les situations, se mêlent conformisme inconscient et respect conscient des normes. Prenons plutôt le parti d'accepter que les normes dont nous allons parler sont connues comme telles ou sont rapidement accessibles à notre conscience comme telles. Elles n'en sont pas moins contraignantes, soit qu'elles s'imposent par la force soit que tout le corps social nous pousse à les accepter et qu'elles participent au système de valeurs de notre groupe. Notre consentement est gradué selon la légitimité que nous accordons à ces obligations. Saluer en entrant dans une salle d'attente, laisser sa chaise à une personne âgée, ne pas parler des sujets qui fâchent, tout ce qui relève de la politesse fait partie de ces normes formalisées tout en restant informelles lorsqu'elles sont comparées à des lois et des règlements impératifs. Nous y consentons d'autant plus facilement que cela nous évite les remarques désagréables, les feux de la stigmatisation et puis surtout nous y trouvons parfois l'avantage de la réciprocité. Bien que les normes vestimentaires, culinaires, relationnelles, cultuelles, artistiques, morales, économiques, éducatives, réglementaires, légales fassent système et qu'elles forment un tout, cela n'empêche pas de les hiérarchiser. Plus l'échelon hiérarchique est élevé moins le consentement, au sens de libre choix, semble de mise. Ce paradoxe pourrait s'expliquer par les conséquences que le défaut d'acceptation aurait. Alors revenons à la norme de réciprocité, seule norme universelle si l'on excepte le tabou de l'inceste. Dans leurs travaux sur le don, Marcel Mauss (1923-1924), Claude Lévi-Strauss (1949) et Jacques Godbout (1992) décryptent les mécanismes sousjacents à ce rituel. Bien sûr il est question de consentement mais surtout d'obligation, de donner, de recevoir, de rendre. Celui qui donne, qui entame un essai de relation se met en danger puisqu'il s'expose à un refus. Seulement un tel refus équivaut à une déclaration de guerre au sens propre ou figuré selon la population considérée. Finalement, il s'agit moins de consentir que de choisir entre une réciprocité positive et une réciprocité négative. 


\section{Qui veille?}

\section{La loi et les juges}

Moins largement partagé que la norme de réciprocité, si nous acceptons de dire que nous ne sommes pas à «La fin de l'histoire » (Fukuyama, 1993), au sommet de notre pyramide des normes : l'individualisme. Il irrigue le contrat social que nous devons respecter sans le signer. Nous naissons citoyens avec, à la clef, un ensemble de droits et de devoirs que probablement nos aînés ont accepté mais dont nous héritons sans notre consentement. Bien que la frontière entre les différents types de normes soit difficile à tracer, leur force obligatoire variant selon les individus et les groupes sociaux, les normes légales sont d'une autre nature que les normes morales, les usages ou les modes. A chaque fois cependant que nous essayons de construire une typologie des normes, l'exception surgit, pratiquement incontournable ; ici : la question des usages car certains usages s'imposent à la loi. Alors, pour plus de clarté, nous admettrons que notre analyse portera davantage sur la règle que sur l'exception qui la confirme. Ainsi, le consentement est encadré par la loi qui protège les individus les uns des autres. C'est dans ce contexte qu'il faut replacer les techniques de manipulation et la naturalisation/ dénaturalisation avec contrat d'action à la clef. L'article 1109 du code civil stipule que : "Il n'y a point de consentement valable si le consentement n'a été donné que par erreur ou s'il a été extorqué par violence ou surpris par dol. ». La loi empêche un trop fort déséquilibre entre les citoyens mais, dit-on, ne protège pas les imbéciles. En revanche, les faibles d'esprit, clairement identifiés comme tels et considérés comme des incapables majeurs, bénéficient de la protection de la loi. Si nous examinons avec attention cet article de loi, il ne fait pas de doute que son interprétation est mal aisée : comment définir l'erreur, la violence ou encore le dol ? Même si les articles suivant l'article 1109 éclairent la question, la jurisprudence n'est pas définitivement fixée, les tribunaux s'interrogent sur la notion de consentement travaillée sans cesse par les courants idéologiques du moment. Deux écoles s'affrontent, l'une défendant la liberté contractuelle et l'autre l'égalité. École de l'autonomie de la volonté contre doctrine du solidarisme contractuel (Grynbaum et Nicod, 2004). Soit l'on considère que les co-contractants sont des hommes libres et égaux et qu'ils sont en mesure de décider librement et en conscience de la teneur du contrat, soit on affirme que dans les faits les hommes ne sont ni libres ni égaux et qu'il faut veiller absolument à 
ce que le consentement ne soit pas vicié. Il semble que le solidarisme fasse son chemin et teinte légèrement les décisions de la Cour de cassation qui veille à la bonne foi des parties et à l'équilibre de leurs droits dans la formation des contrats. Tous les contrats ne sont pas concernés, loin de là. Les belles promesses tenues en vue de la conclusion d'un contrat de mariage par exemple n'engagent que ceux qui les croient ! Ce recul (très relatif) de l'autonomie de la volonté paraît paradoxal dans une époque marquée par la distension du lien social et l'éclatement des solidarités. L'individualisme exacerbé se mue en égoïsme. Les juges en viendraient-ils par réaction à tenter de moraliser les rapports humains alors que cela n'entre pas dans leurs attributions ? Seraient-ils le dernier rempart contre la barbarie des rapports de force ? Pour faire simple et distinguer le droit de la morale, disons que la loi sépare le juste et l'injuste et la morale, le bien et le mal. La séparation entre droit et morale est malaisée, elle n'existe pas dans les théocraties, mais, même dans les états de droit, les éléments distinctifs ne font pas l'unanimité. La morale est affaire de conscience individuelle, elle est imprécise et se loge aussi là où le droit n'intervient pas : par exemple dans les intentions et les mensonges qui ne causent pas de préjudice sanctionné par la loi. Le Droit est généralement précis, il s'applique à tous et gère les comportements pour maintenir les équilibres sociaux. Il se heurte à la morale lorsqu'il permet par exemple au voleur de devenir propriétaire d'un bien qu'il a volé, ce que la morale réprouve, et s'intéresse à des comportements que la morale ignore comme l'interdiction de rouler à droite. Outre que le mélange du droit et de la morale, sans l'aval préalable du parlement, soit critiquable car les juges n'ont pas à appliquer la morale mais le droit uniquement, cette évolution jurisprudentielle pourrait s'expliquer autrement. Les connaissances sur l'homme augmentant, les juges savent désormais que nous sommes tous susceptibles d'être manipulés et donc pas toujours capables d'exercer l'autonomie de notre volonté. Ajoutons à cela l'augmentation du nombre de sujets considérés comme capables majeurs. En effet, les femmes sont sorties de leur statut d'incapables majeures depuis 1945 seulement et peuvent ouvrir un compte bancaire sans l'autorisation de leur mari depuis 1970. Enfin, la majorité est passée de 21 ans à 18 ans, multipliant ainsi les présumés capables majeurs. Autant de sujets capables légalement mais démunis face à leur interlocuteur lorsque ce dernier souhaite profiter de leur faiblesse. Sans machisme ne mauvais aloi, l'équilibre d'un contrat tient pour une large part à un rapport de force informel : nous acceptons de contracter dans le cadre légal car il ne fait pas de doute qu'en cas de non respect 
du texte, le rapport de force reprend ses droits. Quel rapport de force avec une femme âgée, un jeune adulte ? Les juges ne sont pas dupes de ces réalités et certains tentent de les intégrer dans leur jurisprudence. Il est donc moins question de morale que de prise en compte de l'effectivité du changement des relations sociales. Le juge doit rééquilibrer les rapports de force en matière contractuelle pour plus de justice. La justice peut paraître ou non conservatrice si le maintien des équilibres sociaux s'entend d'équilibres anciens et du maintien de la hiérarchie des classes sociales ou d'équilibres démocratiques où les citoyens sont considérés comme égaux. Il ne devrait y avoir qu'une justice possible mais force est de constater que le pot de fer gagne souvent contre le pot de terre. Notre évaluateur de personnalité, promoteur de la soumission librement consentie pourrait trouver grâce auprès d'une justice conservatrice qui considère que le «bon père de famille », c'est-à-dire celui qui se comporte conformément aux attentes de la société, est davantage issu des catégories socioprofessionnelles favorisées et que l'ignorant mal élevé se recrute dans les milieux défavorisés. Alors manipuler un peu ne serait qu'un peu de paternalisme de bon aloi.

\section{La morale ou l'éthique}

Tout cela ne permet toutefois pas d'écarter les liens actuels du consentement et de la morale. Nous pourrions penser en première approche que la morale veille forcément à la liberté du consentement. La morale comme garant du consentement éclairé et de la liberté de nos consciences, cela va de soi. Pourtant, dans un contexte d'anomie généralisée, la morale perd de sa vertu et devient la bonne cause et finalement la fin qui justifie les moyens si c'est pour la bonne cause. Les grands idéaux ont cédé le pas au pragmatisme puisque le modèle dominant n'est plus celui des Lumières. Cependant, en même temps, l'éthique revient sur le devant de la scène, comme pour mieux accompagner le développement durable, relevant d'une même logique, d'une même priorité. Aux désordres d'un libéralisme aveugle répond l'impérieuse nécessité de revenir à des règles morales rebaptisées éthiques. Cette nouvelle appellation n'est peut-être pas simplement un rajeunissement et une laïcisation d'un terme alourdi par ses liens profonds et anciens avec la religion. Plus légère que la morale, l'éthique permettrait de joindre bonne cause et efficacité et pourrait naître du mariage de l'anomie et du recul de la démocratie. 


\section{Logique de résultat}

L'éthique est la fille de l'ère post-industrielle. Passant d'une logique de moyens à une logique de résultats, les entreprises avancent au rythme de la société toute entière. L'ère industrielle a été marquée par une logique de moyens : l'accent était davantage porté sur les moyens que sur les résultats. L'avènement de l'ère post-industrielle a inversé la logique : une liberté de choix des moyens plus grande en contrepartie d'exigences accrues en matière de performance et de résultats. Cette logique de résultat a irrigué toutes les organisations jusqu'à la fonction publique par une loi intitulée LOLF (Loi Organique relative aux Lois de Finances) de 2001. Nous sommes sommés d'obtenir des résultats en inventant éventuellement les moyens pour y parvenir. Il faut être efficace donc, manipuler un peu entre parfaitement dans cette logique, pourvu que l'on obtienne les résultats attendus. L'ambiguïté tient à la définition de ce qu'est un moyen. Les moyens humains font partie du nouveau modèle tout comme le style de direction des personnels où la psychologie tient une place prépondérante. N'y a-t-il pas toujours manipulation?

\section{Conclusion}

Il semble que tout concourre à accepter les entorses à la liberté de notre consentement. Il y a péril en la demeure et toute opposition de principe fait figure d'irénisme, de naïveté ou d'inconscience. La compréhension des mécanismes cognitifs engendre la tentation de manipuler, les études sur l'altération inconsciente du consentement sont réexaminées, appliquées à d'autres terrains. Il n'est plus temps de mettre en garde nos contemporains sur la manière dont leur acceptation peut être viciée. Il suffit de se convaincre que le consentement n'a finalement jamais existé. La chose est aisée : nous avons été formatés par notre famille, son église, les gouvernants, l'école « Car il faut que tous sachent lire pour que ce dernier [le Pouvoir] puisse dire : nul n'est censé ignorer la loi. » (Claude Lévi-Strauss, 1955, p. 355)... Le Pouvoir au sens que Maurice Duverger (1975) lui a donné, c'est-à-dire les faiseurs d'opinion, est comptable de l'avenir et finalement la morale impose une entorse au respect de la liberté individuelle, du consentement éclairé. Nous sommes à des années lumière de l'invention de la démocratie et de ses défenseurs : "Cléon défendait la démocratie en soutenant que l'intérêt de la cité était mieux défendu par cette majorité (plèthos) de 
gens ordinaires, ignorants certes, mais modérés et sensés (sôphrones), [...] 》 (Alain Fouchard, 1997, p. 211). Dans une société de l'information et de la connaissance, l'ignorance est un défaut rédhibitoire. L'éthique exige une attitude responsable, individualiste en somme. Les citoyens ordinaires sont irresponsables et moralement il n'est pas possible de les en blâmer : ils subissent de plein fouet les abus de la société de consommation dans laquelle ils ont été plongés à leur corps souvent défendant. Il est charitable et par là moral de les sortir de cet état d'hypnose en les manipulant pour obtenir un consentement qu'ils auraient donné s'ils avaient eu les idées claires. La morale est sauve mais sa définition mérite probablement une redéfinition. Qui fixera la limite de ce qui relève de la bonne cause ? La crise écologique comme toutes les crises est propice au recul de notre consentement éclairé.

\section{Références bibliographiques}

BEAUVOIS Jean-Léon \& JOULE Robert Vincent, 1987, Petit traité de manipulation à l'usage des honnêtes gens, Grenoble, Presses Universitaires de Grenoble.

DURKHEIM Émile, 1888, "Cours de science sociale. Leçon d'ouverture », Revue internationale de l'enseignement, XV, p. 23-48.

DUVERGER Maurice, 1975, Institutions politiques et Droit Constitutionnel 1/ les grands systèmes politiques, Paris, Presses Universitaires de France.

FOUCHARD Alain, 1997, Aristocratie et démocratie. Idéologie et sociétés en Grèce ancienne, Besançon, Annales littéraires de l'Université de Franche-Comté.

FUKUYAMA Francis, 1993, La fin de l'histoire et le dernier homme, Paris, Flammarion.

GRYNBAUM Luc, NICOD Marc (éd.), 2004, Le solidarisme contractuel, Paris, Economica.

GODBOUT Jacques, 1992, L'esprit du don, Paris, La découverte.

JOULE Robert-Vincent, BEAUVOIS Jean-Léon, 1987, Petit traité de manipulation à l'usage des honnêtes gens, Grenoble, Presses Universitaires de Grenoble.

JOULE Robert-Vincent, BEAUVOIS Jean-Léon, 1998, La soumission librement consentie, Paris, Presses Universitaires de France.

KIESLER Charles, 1971, The psycology of commitment. Experiments linking behavior to belief, New York, Academic Press.

LA PIERE Richard, 1934, «Attitudes vs actions », Social forces.

LÉVI-STRAUSS Claude, 1949, Les structures élémentaires de la parenté, Paris, Presses Universitaires de France.

LÉVI-STRAUSS Claude, 1955, Tristes tropiques, Paris, Plon.

LEWIN Kurt, 1947, « Group decision and social change », in Newcomb Theodore et Hartley Eugene (éd.), Readings in social psychology, New York, Holt.

MAUSS Marcel, 1923-1924, «Essai sur le don. Forme et raison de l'échange dans les sociétés primitives », l'Année Sociologique, seconde série.

SARTRE Jean-Paul, 1996, L'existentialisme est un humanisme, Paris, Gallimard, (1 $1^{\text {̀̀re }}$ éd. 1945) 